\title{
A Simple Approach of Determination of the Crystallographic Orientation: Applications and Accuracy
}

\author{
S.C. Wang* and M.J. Starink
}

Materials Research Group, School of Engineering Sciences, The University of Southampton, SO17 1BJ, England

Key words: Beam direction, crystallographic orientation, Kikuchi lines

\begin{abstract}
A simple analytical solution for the crystallographic orientation has been described. This method is based on one indexed Kikuchi pair in a known zone rather than the corresponding diffraction spots. The accuracy of this method is shown to be better than $0.1^{\circ}$ even in the cases where a zone axis is deviated by a large angle (e.g.1 $\left.10^{\circ}\right)$ form the centre of the beam direction. This approach simplifies experiments since only one pair of Kikuchi lines and a zone axis are needed, and is especially suited when it is difficult or cumbersome to resolve a second pair of Kikuchi lines with sufficient accuracy.
\end{abstract}

\footnotetext{
* To whom all correspondence should be addressed.

E-mail address: wangs@soton.ac.uk
} 


\section{Introduction}

The knowledge of crystallographic orientation is of considerable importance such as in the study of the defects of grains, grain boundaries and heterophase interfaces. Determination of the orientation using TEM has the outstanding advantage that the orientation and image of the microstructure of a specimen can be recorded at the same time with a high spatial resolution. All the accurate methods used to determine the beam direction make use of Kikuchi lines combined with the corresponding diffraction spots. For example, Ryder and Pitsch (1968), Thomas and Goringe (1979) proposed a geometrical method to solve the orientation, in which at least three strong spots and three pairs of Kikuchi lines which were sharp and well defined, not belonging to the same zone were needed in the diffraction pattern. A relatively simple method was proposed by Pumphrey and Bowkett (1970), in which two Kikuchi bands in addition to the diffraction spots were required. Other methods such as Heimendahl et al (1964), and Ball (1981) make use of at least two or three independent pairs of Kikuchi lines and diffraction spots. These methods, however, have a similar weakness since the clarity of Kikuchi lines and diffraction spots exist in a slight different range of thickness of a specimen. The convergent-beam-electron-diffraction (CBED) technique, which is available for the modern electron microscopes, may be used to obtain high-quality Kikuchi patterns in a large range of thickness for most specimens. In CBED patterns, however, it may be difficult to identify the correspondence between the Kikuchi lines and diffraction spots as the latter are generally blurred or difficult to resolve. Therefore, application of these methods is limited. To overcome such weakness, Helfmeier and Feller-Kniepmeier (1977) proposed a method based on two pairs of Kikuchi lines without consideration of diffraction spots. This method, however, was found difficult to apply as the signs of two pairs of Kikuchi lines as well as directions of lines perpendicular to the Kikuchi lines must be known.

As the automatic indexing of backscattered Kikuchi bands (BSKB) has already been applied commercially on SEM (e.g. HKL Channel+ software, Oxford Instruments OPAL), a number of recent studies are focused on fully 'automatic' indexing of transmission Kikuchi bands (TMKB) on TEM (e.g. Schwarzer 1997, Morawiec 1999 and Zaefferer 2000). The indexing is based on the comparison of measured interplanar angles and interplanar spacings with theoretical values calculated for the actual crystal structure (Schwarzer 1997). However, application of automatic indexing on TMKB is more difficult than on BSKB, because of the generally poorer quality of TMKB, which depends not only the surface strains (similar to BSKB in SEM) but also the specimen thickness. According to Schwarzer (1997), its accuracy by use of automatic indexing was proved up to $0.5^{\circ}$, whereas it is only $0.1^{\circ}$ by manual indexing. Thus, manual indexing is still widely used for accurate determination of crystallographic orientations, defect simulations, etc.

In this paper, a new method to determine the beam direction is proposed. There are some advantages in using this method. Firstly, it can be used to analyse patterns which cannot be analysed using other methods, such as patterns where the diffraction spots are blurred or can not be distinguished. Secondly, in general at least one zone and a pair of Kikuchi lines can be recorded in a CBED pattern using a low camera length, thus it is not necessary to search for special orientations where at least two distinct Kikuchi bands can be indexed. It may therefore simplify the procedure to determine the boundary misorientations and heterophase orientation relationships in which usually three pairs of orientations are needed. Thirdly, it can be applied to the case where a zone axis is deviated by a large angle (e.g. $10^{\circ}$ ) from the beam direction.

\section{The principle of the method}


Generally at least one zone can be included in a diffraction pattern recorded using a low camera length. Assuming that the vector parallel to the axis of a zone $\mathrm{A}$ is $\mathbf{u}_{\mathrm{A}}$ and the vector parallel to the beam direction is $\mathbf{u}_{\mathrm{O}}$, then a line, which is perpendicular to $\mathbf{u}_{\mathrm{A}}$ and lies in the plane containing $\mathbf{u}_{\mathrm{A}}$ and $\mathbf{u}_{\mathrm{O}}$, can be obtained. This corresponding vector is designated $\mathbf{k}$. Fig. 1a illustrates the formation of zone A related to the Ewald sphere. $O_{1}$ is the centre of the Ewald sphere, $O$ lies on the sphere. $\mathbf{u}_{\mathrm{O}}$ is parallel to $O O_{1}$, and $\mathbf{u}_{\mathrm{A}}$ is parallel to $A O_{1}$. A pair of Kikuchi lines can be formed by the intersection of the Kossel cones (illustrated by the dotted lines $O_{1} B$ and $O_{1} C$ in two dimension) and the Ewald sphere. $B$ and $C$ are the positions of a pair of Kikuchi lines viewed end-on, and $A$ is the central position. The distance of the Kikuchi band away from the beam centre recorded in the plane perpendicular to $\mathbf{u}_{\mathrm{O}}$ is $A_{2} O$, and the corresponding distance in the plane perpendicular to $\mathbf{u}_{\mathrm{A}}$ is $A O_{2}$. Assuming that in reciprocal space $A O_{2}=m, A_{2} O=m^{\prime}$ and $A O_{1}=O O_{1}=1 / \lambda$ (where $\lambda$ is the electron wave length), then the following equation is obeyed

$$
\mathbf{u}_{\mathrm{O}}=\frac{1}{\sqrt{1+(\lambda \cdot m)^{2}}} \cdot \frac{\mathbf{u}_{\mathrm{A}}}{\left|\mathbf{u}_{\mathrm{A}}\right|}-\frac{\lambda \cdot m}{\sqrt{1+(\lambda \cdot m)^{2}}} \cdot \frac{\mathbf{k}}{|\mathbf{k}|}
$$

The top diagram in Fig. $1 \mathrm{~b}$ shows the relations among $\mathbf{g}, \mathbf{k}_{2}$ and $\mathbf{k}$ in the plane perpendicular to $\mathbf{u}_{\mathrm{A}}$, as seen from Fig. 1a, the distance of $\mathrm{AO}_{2}$ is $m$, thus,

$$
\mathbf{k}=\frac{m_{1}}{\sqrt{m_{1}^{2}+m_{2}^{2}}} \cdot \frac{\mathbf{g}}{|\mathbf{g}|}+\frac{m_{2}}{\sqrt{m_{1}^{2}+m_{2}^{2}}} \cdot \frac{\mathbf{k}_{2}}{\left|\mathbf{k}_{2}\right|}
$$

where $\mathbf{g}$ is a reciprocal lattice vector perpendicular to a pair of Kikuchi lines which lie perpendicular to $\mathbf{u}_{\mathrm{A}}$, and $\mathbf{k}_{2}$ is the vector parallel to a line which lies perpendicular to $\mathbf{g}$ and $\mathbf{u}_{\mathrm{A}}, m_{1}$ and $m_{2}$ are the components of $m$ resolved parallel to $\mathbf{g}$ and $\mathbf{k}_{2}$, respectively, then

$$
\mathbf{k}_{2}=\mathbf{g} \times \mathbf{u}_{\mathrm{A}}
$$

Combining equations (1 3), the beam direction can be solved if $m_{1}, m_{2}$ and $m$ are known. However, these data cannot be obtained directly because TEM measurements are carried out on the plane perpendicular to $\mathbf{u}_{\mathrm{O}}$ rather than on the plane perpendicular to $\mathbf{u}_{\mathrm{A}}$.

If $\mathbf{k}^{\prime}$ and $m^{\prime}$ represent the projected direction and distance in the plane perpendicular to $\mathbf{u}_{\mathrm{O}}$ corresponding to $\mathbf{k}$ and $m$ in the plane perpendicular to $\mathbf{u}_{\mathrm{A}}$, then the following relation is satisfied from Fig. 1a,

$$
m=\frac{m^{\prime}}{\sqrt{1-\left(\lambda \cdot m^{\prime}\right)^{2}}}
$$

where $\psi$ is a angle between $\mathbf{u}_{\mathrm{O}}$ and $\mathbf{u}_{\mathrm{A}}$ or between $\mathbf{k}$ and $\mathbf{k}^{\prime}$.

Similarly, if $\mathbf{k}_{1}^{\prime}$ and $\mathbf{k}_{2}^{\prime}$ are vectors parallel to the projections of $\mathbf{g}$ and $\mathbf{k}_{2}$, respectively, perpendicular to $\mathbf{u}_{\mathrm{O}}$ and having magnitudes $m_{1}^{\prime}$ and $m_{2}^{\prime}$, respectively, then the angles $\psi_{1}$ and $\psi_{2}$ corresponding to $\mathbf{g}^{\wedge} \mathbf{k}_{1}^{\prime}$ and $\mathbf{k}_{2} \wedge \mathbf{k}_{2}^{\prime}$, respectively (see Fig. 1c), are given by

$$
m_{1}=\frac{m_{1}^{\prime}}{\sqrt{1-\left(\lambda \cdot m_{1}^{\prime}\right)^{2}}}
$$


and

$$
m_{2}=\frac{m_{2}^{\prime}}{\sqrt{1-\left(\lambda \cdot m_{2}^{\prime}\right)^{2}}}
$$

It is worth noting that although $\mathbf{g}$ is perpendicular to $\mathbf{k}_{2}$ in the plane normal to $\mathbf{u}_{\mathrm{A}}$, it does not mean that their projected lines $\mathbf{k}_{1}^{\prime}$ and $\mathbf{k}_{2}^{\prime}$ in the plane perpendicular to $\mathbf{u}_{\mathrm{O}}$ are still perpendicular. The bottom of Fig. $1 b$ illustrates the relation between $\mathbf{k}_{1}^{\prime}$ and $\mathbf{k}_{2}^{\prime}$ in the plane perpendicular to $\mathbf{u}_{\mathrm{O}}$. Thus, $m_{1}^{\prime}$ and $m_{2}^{\prime}$ cannot be obtained directly from a CBED pattern unless $\phi$ is known. As shown in Fig. $1 \mathrm{~b}$, only the components $\Delta_{1}$ and $\Delta_{2}$ (perpendicular to and parallel to the projected Kikuchi lines) can be given. Their relations are as follows

$$
\begin{aligned}
& m_{1}^{\prime}=\frac{\Delta_{1}}{L \cdot \lambda \cdot \sin (\phi)} \\
& m_{2}^{\prime}=\frac{\Delta_{2}-\Delta_{1} c \tan (\phi)}{L \cdot \lambda}
\end{aligned}
$$

and

$$
m^{\prime}=\frac{\sqrt{\left(\Delta_{1}\right)^{2}+\left(\Delta_{2}\right)^{2}}}{L \cdot \lambda}
$$

where $L$ is the effective camera length. From the appendix, $\phi$ may be given by equation (A5), then $m_{1}^{\prime}$ and $m_{2}^{\prime}$ can be expressed by $\Delta_{1}$ and $\Delta_{2}$.

With the above procedure, once the experimental measurements of $\Delta_{1}, \Delta_{2}$ and the effective camera length $L$ are obtained, the beam direction can be calculated,

$$
\mathbf{u}_{0}=\frac{\mathbf{u}_{\mathrm{A}}}{\left|\mathbf{u}_{\mathrm{A}}\right|}-\frac{1}{\sqrt{\frac{L^{2}}{\left(\Delta_{1}\right)^{2}+\left(\Delta_{2}\right)^{2}}-1}}\left(\frac{1}{\sqrt{1+\mathrm{n}}} \frac{\mathbf{g}}{|\mathbf{g}|}+\frac{1}{\sqrt{1+1 / \mathrm{n}}} \frac{\mathbf{k}_{2}}{\left|\mathbf{k}_{2}\right|}\right)
$$

where

$$
\mathrm{n}=\left(\frac{L^{4} \cdot\left(L^{2}-\left(\Delta_{1}\right)^{2}-\left(\Delta_{2}\right)^{2}\right)}{\left(\Delta_{1}\right)^{2} \cdot\left(L^{2}-\left(\Delta_{1}\right)^{2}\right) \cdot\left(L^{2}-\left(\Delta_{2}\right)^{2}\right)}-1\right) /\left(\frac{L^{4} \cdot\left(L^{2}-\left(\Delta_{1}\right)^{2}-\left(\Delta_{2}\right)^{2}\right)}{\left(\Delta_{2}\right)^{2} \cdot\left(L \cdot \sqrt{L^{2}-\left(\Delta_{1}\right)^{2}-\left(\Delta_{2}\right)^{2}}+\left(\Delta_{1}\right)^{2}\right)^{2}}-1\right)
$$

The equation (6) gives the resolution for the beam direction. However, the equation for factor $\mathrm{n}$ is very complex and possible measurement errors introduced are, therefore, not easy to be determined. However if $\Delta_{1}=0$, then equation (6) can be simplified as

$$
\mathbf{u}_{0}=\frac{\mathbf{u}_{\mathrm{A}}}{\left|\mathbf{u}_{\mathrm{A}}\right|}-\frac{1}{\sqrt{\frac{L^{2}}{\left(\Delta_{2}\right)^{2}}-1}} \cdot \frac{\mathbf{k}_{2}}{\left|\mathbf{k}_{2}\right|}
$$

Then $\Delta_{1} \cdot \frac{\mathbf{g}}{|\mathbf{g}|}+\Delta_{2} \cdot \frac{\mathbf{k}_{2}}{\left|\mathbf{k}_{2}\right|}$ and $\sqrt{\left(\Delta_{1}\right)^{2}+\left(\Delta_{2}\right)^{2}}$ replace $\mathbf{k}_{2}$ and $\Delta_{2}$, and equation (7) becomes 
where

$$
\mathbf{u}_{0}=\frac{\mathbf{u}_{\mathrm{A}}}{\left|\mathbf{u}_{\mathrm{A}}\right|}-\frac{1}{\sqrt{\frac{L^{2}}{\left(\Delta_{1}\right)^{2}+\left(\Delta_{2}\right)^{2}}-1}} \cdot \frac{\Delta_{1} \cdot \frac{\mathbf{g}}{|\mathbf{g}|}+\Delta_{2} \cdot \frac{\mathbf{k}_{2}}{\left|\mathbf{k}_{2}\right|}}{\left|\Delta_{1} \cdot \frac{\mathbf{g}}{|\mathbf{g}|}+\Delta_{2} \cdot \frac{\mathbf{k}_{2} \mid}{\left|\mathbf{k}_{2}\right|}\right|}
$$

or

$$
\mathbf{k}_{2}=\mathbf{g} \times \mathbf{u}_{\mathrm{A}} \text { in case of Fig. 1b }
$$

Equation (8) has also been proven, experimentally, to given the same resolution as equation (6) (Wang 1998).

\section{Applications and Accuracy}

To resolve the beam direction in Equation (8), five steps are suggested to follow: (1) experimental measurements of $\Delta_{1}, \Delta_{2}$; (2) determination of the zone axis $\mathbf{u}_{\mathrm{A}}$ based on the diffraction or Kikuchi pattern; (3) determination of one Kikuchi band $\mathbf{g}$; (4) choice of direction $\mathbf{k}_{2}$ by drawing a direction line from the known zone to the required zones/ beam centre; (5) calculation of the effective camera length $L$. There are two approaches to determine $L$ : one is $L=R \cdot d / \lambda$, where $R$ is distance between one pair of Kikuchi lines in reciprocal space and $d$ is the spacing of the corresponding plane. The second is $L=\Delta / \sin \varphi$, where $\Delta$ and $\varphi$ are distance and angle between two known zones. It is recommended to use the second method if more than two zones of Kikuchi patterns are recorded.

The validity of this method can be confirmed by analysing patterns obtained at orientations where three or more zone axes were recorded using TEM. Fig. 2a shows an example of such a CBED pattern from hexagonal $\mathrm{Ti}$ : the arrow indicates the centre of electron beam. Fig. $2 \mathrm{~b}$ shows the schematic diagram of Fig. 2a. Four zone axes were determined as: Zone B, [2 203 ]; Zone C, $[7, \overline{8}, 1,12]$, Zone D, $[8, \overline{7}, \overline{1}, 12]$ and Zone $\mathrm{P},[1 \overline{1} 02]$. Assuming that the lattice parameters are $\mathrm{a}=$ $0.29511 \mathrm{~nm}, \mathrm{c}=0.46843 \mathrm{~nm}$, the angle between $\mathrm{B}, \mathrm{C}$, and $\mathrm{D}$ away from $\mathrm{P}$ are calculated: $\varphi_{\mathrm{BP}}=$ $7.418^{\circ}, \varphi_{\mathrm{CP}}=6.785^{\circ}$ and $\varphi_{\mathrm{DP}}=6.785^{\circ}$. The effective camera length is 761.7(4) $\mathrm{mm}$, and hence the distances between two zones are: $\Delta_{\mathrm{BP}}=L \sin \left(\varphi_{\mathrm{BP}}\right)=98.35 \mathrm{~mm} ; \Delta_{\mathrm{CP}}=L \sin \left(\varphi_{\mathrm{CP}}\right)=90 \mathrm{~mm} ; \Delta_{\mathrm{DP}}=L$ $\sin \left(\varphi_{\mathrm{DP}}\right)=90 \mathrm{~mm}$. Fig. $3 \mathrm{c}$ and the column 5 in Table 1 show the relations among $\mathbf{u}_{\mathrm{A}}\left(=\mathbf{u}_{\mathrm{B}}, \mathbf{u}_{\mathrm{C}}\right.$ or $\left.\mathbf{u}_{\mathrm{D}}\right)$, $\mathbf{g}$ and $\mathbf{k}_{2}$. When these data were input in equation (8) and all the solutions for zone $\mathrm{P}$ gave $\mathbf{u}_{\mathrm{P}}=$ [ $1 \overline{1}$ 102] (see Table 1 for detail), which clearly proved the validity of equation (8).

Table 1. Calculation of zone axis $\mathbf{P}$ from equations (8) based on different $\mathbf{g}$ and known zone axes.

\begin{tabular}{|c|c|c|c|c|c|c|}
\hline $\begin{array}{l}\text { Known } \\
\text { zone } \mathbf{u}_{\mathrm{A}}\end{array}$ & g & $\Delta 1(\mathrm{~mm})$ & $\Delta 2(\mathrm{~mm})$ & $\mathbf{k}_{2}$ & Illustration & $\begin{array}{c}\text { Solution for } \\
\text { Zone P }\end{array}$ \\
\hline \multirow{3}{*}{ B } & $\overline{1} \overline{1} 20$ & 0 & 98.35 & $\mathbf{u}_{\mathrm{B}} \times \mathbf{g}$ & Fig.3c (i) & {$\left[\begin{array}{lll}1 & \overline{1} & 02\end{array}\right]$} \\
\hline & $1 \overline{2} 1 \overline{2}$ & 89.11 & 41.60 & $\mathbf{u}_{\mathrm{B}} \times \mathbf{g}$ & Fig.3c (ii) & {$\left[\begin{array}{lll}1 & \overline{1} & 02\end{array}\right]$} \\
\hline & $2 \overline{1} \overline{1} \overline{2}$ & 41.60 & 89.11 & $\mathbf{g} \times \mathbf{u}_{\mathrm{B}}$ & Fig.3c (iii) & {$\left[\begin{array}{lll}1 & \overline{1} & 02\end{array}\right]$} \\
\hline \multirow{2}{*}{$\mathrm{C}$} & $\overline{2} 021$ & 0 & 90 & $\mathbf{u}_{\mathrm{C}} \times \mathbf{g}$ & Fig.3c (iv) & {$\left[\begin{array}{lll}1 & \overline{1} & 02\end{array}\right]$} \\
\hline & $1 \overline{2} 1 \overline{2}$ & 89.11 & 12.63 & $\mathbf{g} \times \mathbf{u}_{\mathrm{C}}$ & Fig.3c (v) & {$\left[\begin{array}{lll}1 & \overline{1} & 02\end{array}\right]$} \\
\hline \multirow{2}{*}{ D } & $02 \overline{2} 1$ & 0 & 90 & $\mathbf{g} \times \mathbf{u}_{\mathrm{D}}$ & Fig.3c (vi) & {$\left[\begin{array}{lll}1 & \overline{1} & 02\end{array}\right]$} \\
\hline & $2 \overline{1} \overline{1} \overline{2}$ & 89.11 & 12.63 & $\mathbf{u}_{\mathrm{D}} \times \mathbf{g}$ & Fig.3c (vii) & {$\left[\begin{array}{lll}1 & \overline{1} & 02\end{array}\right]$} \\
\hline
\end{tabular}
The measurements were carried out at an effective camera length $L=761.7 \mathrm{~mm}$. 
The error for solution of the direction in equation (8) may be estimated by assuming that the maximum errors by experimental measurements of $\Delta_{1}, \Delta_{2}$ are $\pm 0.5 \mathrm{~mm}$ and the error for the effective camera length $L$ is $\pm 2.5 \mathrm{~mm}$. As shown in equation (8), $\Delta_{1}$ and $\Delta_{2}$ have the opposite effects on $\mathbf{u}_{\mathrm{O}}$ compared to $L$, thus opposite values are chosen. The details are shown in Table 2 , which indicates that the maximum errors are indeed less than $0.1^{\circ}$. Similarly, a lot of data are available to solve the beam direction of spot $\mathrm{O}$ as shown in Table 3. The resultant $\mathbf{u}_{\mathrm{O}}$ are very close to each other and the remaining small deviations (less than $0.03^{\circ}$ ) are believed to be caused by the measurements.

Table 2. Zone axes and the beam direction calculated by equations (8). The measurements were carried out at an effective camera length $L=761.7^{ \pm 2.5} \mathrm{~mm}$.

\begin{tabular}{|c|c|c|c|c|c|c|}
\hline $\begin{array}{l}\text { Known } \\
\text { zone } \mathbf{u}_{\mathrm{A}}\end{array}$ & g & $\Delta 1(\mathrm{~mm})$ & $\Delta_{2}(\mathrm{~mm})$ & $L$ & $\mathbf{u}_{\mathrm{P}}$ & $\Delta \mathbf{u}_{\mathrm{P}}^{*}$ \\
\hline \multirow{6}{*}{ B } & \multirow{2}{*}{$\overline{1} \overline{1} 20$} & $0^{+0.5}$ & $98.35^{+0.5}$ & $761.7^{-2.5}$ & {$\left[\begin{array}{llll}.3377 & -.3382 & .0005 & .6776\end{array}\right]$} & $0.074^{\circ}$ \\
\hline & & $0^{-0.5}$ & $98.35^{-0.5}$ & $761.7^{+2.5}$ & {$\left[\begin{array}{llll}.3396 & -.3391 & -.0005 & .6769\end{array}\right]$} & $0.071^{\circ}$ \\
\hline & \multirow[b]{2}{*}{$1 \overline{2} 1 \overline{2}$} & $89.11^{+0.5}$ & $41.60^{+0.5}$ & $761.7^{-2.5}$ & {$\left[\begin{array}{llll}.3377 & -.3380 & .0003 & .6778\end{array}\right]$} & $0.077^{\circ}$ \\
\hline & & $89.11^{-0.5}$ & $41.60^{-0.5}$ & $761.7^{+2.5}$ & {$\left[\begin{array}{llll}.3396 & -.3393 & -.0003 & .6769\end{array}\right]$} & $0.077^{\circ}$ \\
\hline & \multirow{2}{*}{$2 \overline{1} \overline{1} \overline{2}$} & $41.60^{+0.5}$ & $89.11^{+0.5}$ & $761.7^{-2.5}$ & {$\left[\begin{array}{llll}.3380 & -.3377 & -.0003 & .6778\end{array}\right]$} & $0.077^{\circ}$ \\
\hline & & $41.60^{-0.5}$ & $89.11^{-0.5}$ & $761.7^{+2.5}$ & {$\left[\begin{array}{llll}.3393 & -.3396 & .0003 & .6769\end{array}\right]$} & $0.077^{\circ}$ \\
\hline \multirow{4}{*}{$\mathrm{C}$} & \multirow[b]{2}{*}{$\overline{2} 021$} & $0^{+0.5}$ & $90^{+0.5}$ & $761.7^{-2.5}$ & {$\left[\begin{array}{llll}.3388 & -.3379 & -.0009 & .6775\end{array}\right]$} & $0.071^{\circ}$ \\
\hline & & $0^{-0.5}$ & $90^{-0.5}$ & $761.7^{+2.5}$ & {$\left[\begin{array}{llll}.3385 & -.3394 & .0009 & .6771\end{array}\right]$} & $0.071^{\circ}$ \\
\hline & \multirow{2}{*}{$1 \overline{2} 1 \overline{2}$} & $89.11^{+0.5}$ & $12.63^{+0.5}$ & $761.7^{-2.5}$ & {$\left[\begin{array}{llll}.3387 & -.3378 & -.0009 & .6776\end{array}\right]$} & $0.074^{\circ}$ \\
\hline & & $89.11^{-0.5}$ & $12.63^{-0.5}$ & $761.7^{+2.5}$ & {$\left[\begin{array}{llll}.3386 & -.3395 & .0009 & .6771\end{array}\right]$} & $0.071^{\circ}$ \\
\hline \multirow{4}{*}{ D } & \multirow[b]{2}{*}{$02 \overline{2} 1$} & $0^{+0.5}$ & $90^{+0.5}$ & $761.7^{-2.5}$ & {$\left[\begin{array}{llll}.3379 & -.3388 & .0009 & .6775\end{array}\right]$} & $0.071^{\circ}$ \\
\hline & & $0^{-0.5}$ & $90^{-0.5}$ & $761.7^{+2.5}$ & {$\left[\begin{array}{llll}.3394 & -.3385 & -.0009 & .6771\end{array}\right]$} & $0.071^{\circ}$ \\
\hline & \multirow[b]{2}{*}{$2 \overline{1} \overline{1} \overline{2}$} & $89.11^{+0.5}$ & $12.63^{+0.5}$ & $761.7^{-2.5}$ & {$\left[\begin{array}{llll}.3378 & -.3387 & .0009 & .6776\end{array}\right]$} & $0.074^{\circ}$ \\
\hline & & $89.11^{-0.5}$ & $12.63^{-0.5}$ & $761.7^{+2.5}$ & {$\left[\begin{array}{llll}.3395 & -.3386 & -.0009 & .6771\end{array}\right]$} & $0.071^{\circ}$ \\
\hline
\end{tabular}

* The deviation of the calculated $\mathbf{u}_{\mathrm{P}}$ from $\left[\begin{array}{lll}1 & \overline{1} & 0\end{array}\right]$

Table 3. Zone axes and the beam direction calculated by equations (8). The measurements were carried out at an effective camera length $L=760 \mathrm{~mm}$.

\begin{tabular}{|c|c|c|c|c|c|c|}
\hline $\begin{array}{l}\text { Known } \\
\text { zone } \mathbf{u}_{\mathrm{A}}\end{array}$ & g & $\Delta 1(\mathrm{~mm})$ & $\Delta_{2}(\mathrm{~mm})$ & $\mathbf{k}_{2}$ & $\mathbf{u}_{0}$ & $\Delta \mathbf{u}_{0} *$ \\
\hline \multirow{3}{*}{ B } & $\overline{1} \overline{1} 20$ & 1 & 47.5 & $\mathbf{u}_{\mathrm{B}} \times \mathbf{g}$ & {$\left[\begin{array}{llll}.3802 & -.3786 & -.0016 & .6511\end{array}\right]$} & $0.03^{\circ}$ \\
\hline & $1 \overline{2} 1 \overline{2}$ & 43.5 & 19 & $\mathbf{u}_{\mathrm{B}} \times \mathbf{g}$ & {$\left[\begin{array}{llll}.3801 & -.3788 & -.0013 & .6511\end{array}\right]$} & $0.03^{\circ}$ \\
\hline & $2 \overline{1} \overline{1} \overline{2}$ & 42 & 21 & $\mathbf{g} \times \mathbf{u}_{\mathrm{B}}$ & {$\left[\begin{array}{llll}.3805 & -.3792 & -.0013 & .6508\end{array}\right]$} & $0.02^{\circ}$ \\
\hline \multirow{2}{*}{$\mathrm{C}$} & $\overline{2} 021$ & 29.5 & 48 & $\mathbf{u}_{\mathrm{B}} \times \mathbf{g}$ & {$\left[\begin{array}{llll}.3806 & -.3788 & -.0018 & .6509\end{array}\right]$} & $0.02^{\circ}$ \\
\hline & $1 \overline{2} 1 \overline{2}$ & 43.5 & 36 & $\mathbf{g} \times \mathbf{u}_{\mathrm{B}}$ & {$\left[\begin{array}{llll}.3806 & -.3787 & -.0019 & .6509\end{array}\right]$} & $0.03^{\circ}$ \\
\hline \multirow{2}{*}{$\mathrm{D}$} & $02 \overline{2} 1$ & 27 & 46 & $\mathbf{g} \times \mathbf{u}_{\mathrm{B}}$ & {$\left[\begin{array}{llll}.3808 & -.3791 & -.0017 & .6507\end{array}\right]$} & $0.03^{\circ}$ \\
\hline & $2 \overline{1} \overline{1} \overline{2}$ & 42 & 33.5 & $\mathbf{u}_{\mathrm{B}} \times \mathbf{g}$ & {$\left[\begin{array}{llll}.3805 & -.3793 & -.0012 & .6508\end{array}\right]$} & $0.02^{\circ}$ \\
\hline
\end{tabular}

* The deviation of $\mathbf{u}_{\mathrm{O}}$ from the mean direction which is [ [ $\left.\begin{array}{lllll}0.3805 & -0.3790 & -0.0015 & 0.6509\end{array}\right]$

The beam direction is a very important parameter because many boundary characteristics are obtained from it. As described in the Introduction, several methods have been proposed to determine the beam directions. The new method used in this study has some advantages over the others. 
Firstly, it can be used to analyse patterns which cannot be analysed using other methods. For example, Fig. 3 shows a CBED pattern near [2 $\overline{4} 23$ ] zone of $\mathrm{Ti}_{5} \mathrm{Si}_{3}$ phase with only one pair of Kikuchi lines resolved. No published approaches can be used easily to solve the beam direction in this case since the corresponding diffraction spots are difficult to distinguish. However, it is easy to determine the beam direction using our method. Assuming that the lattice parameters of $\mathrm{Ti}_{5} \mathrm{Si}_{3}$ are $a$ $=0.7444 \mathrm{~nm}, c=0.5143 \mathrm{~nm}$, the beam is $\mathrm{O}$ indicated by the needle. At the effective camera length $L$ $=440 \mathrm{~mm}, \Delta_{1}$ and $\Delta_{2}$ were measured to be 18.5 and $1 \mathrm{~mm}$, respectively. As the pair of Kikuchi lines relative to the beam direction in Fig. 3 are similar to Fig.1b, i.e. $\mathbf{k}_{2}=\mathbf{g} \times \mathbf{u}_{\mathrm{A}}$, then the beam direction was calculated as $\left[\begin{array}{llll}0.4156 & -0.7717 & 0.3561 & 0.5745\end{array}\right]$ using equation (8) $\left(\mathbf{g}=\overline{1} 010, \mathbf{u}_{\mathrm{A}}=\left[\begin{array}{l}2 \overline{4} 23\end{array}\right)\right.$. The new method has been developed especially for the determination of boundary misorientations from pairs of diffraction patterns where it is usually difficult to have both patterns aligned to contain the two zones of spots which are necessary for the subsequent analysis. This method is also convenient for determination of the foil normal which may deviate far from the zone axis which can be indexed, and for the computer simulation of defects in two beam conditions where in general the beam is 5$10^{\circ}$ away from the low indexed zone. This method has been presented in hexagonal crystals and may apply to any other systems.

\section{Conclusion}

A simple analytical solution (equation 8) for the electron beam has been devised. This solution is based on only one indexed Kikuchi pair in a known zone, disregard of the corresponding diffraction spots. The accuracy of the method is shown experimentally to be better than $0.1^{\circ}$ in the cases where a zone axis is deviated by up to $10^{\circ}$ from the centre of the beam direction, which is comparable to the accuracy of other published methods. Such errors are caused by the experimental measurements rather than by this approach.

\section{ACKNOWLEDGEMENTS}

The authors are grateful to Prof. M Aindow at University of Connecticut for helpful discussions.

\section{References}

Ball, C.J. (1981) Accurate determination of crystallographic orientation from Kikuchi pattern. Phil. Mag. A, 44, 1307-1317

von. Heimendahl M., Bell, W. and Thomas, G. (1964) Applications of Kikuchi line analyses in electron microscopy. J. Applied Physics, 35, 3614-3616

Helfmeier, H. and Feller-Kniepmeier, M. (1977) Analytical determination of the exact primary beam direction from Kikuchi patterns, J. Applied Physics, 48, 3997-3997

Morawiec, A. (1999) Automatic orientation determination from Kikuchi patterns. J. Appl. Cryst. 32, 788-798

Pumphrey, P.H. and Bowkett, K.M. (1970) An accurate method for determining crystallographic orientations by electron diffraction. Phys. Stat. Sol. A, 2, 339-346

Ryder, P.L. and Pitsch, W. (1968) On the accuracy of orientation determination by selected area electron diffraction. Phil. Mag. A 18, 807-816

Schwarzer, R.A. (1997) Advances in crystal orientation mapping with the SEM and TEM. Ultramicroscopy, 67, 19-24

Thomas, G. and Goringe M.J. (1979) Transmission electron microscopy of materials. New York

Wang, S.C. (1998) High angle grain boundary structure in Widmanstätten alfa Ti microstructures. PhD thesis, University of Birmingham

Zaefferer, S. (2000) New developments of computer-aided crystallographic analysis in transmission electron microscopy. J. Appl. Cryst., 33, 10-25 

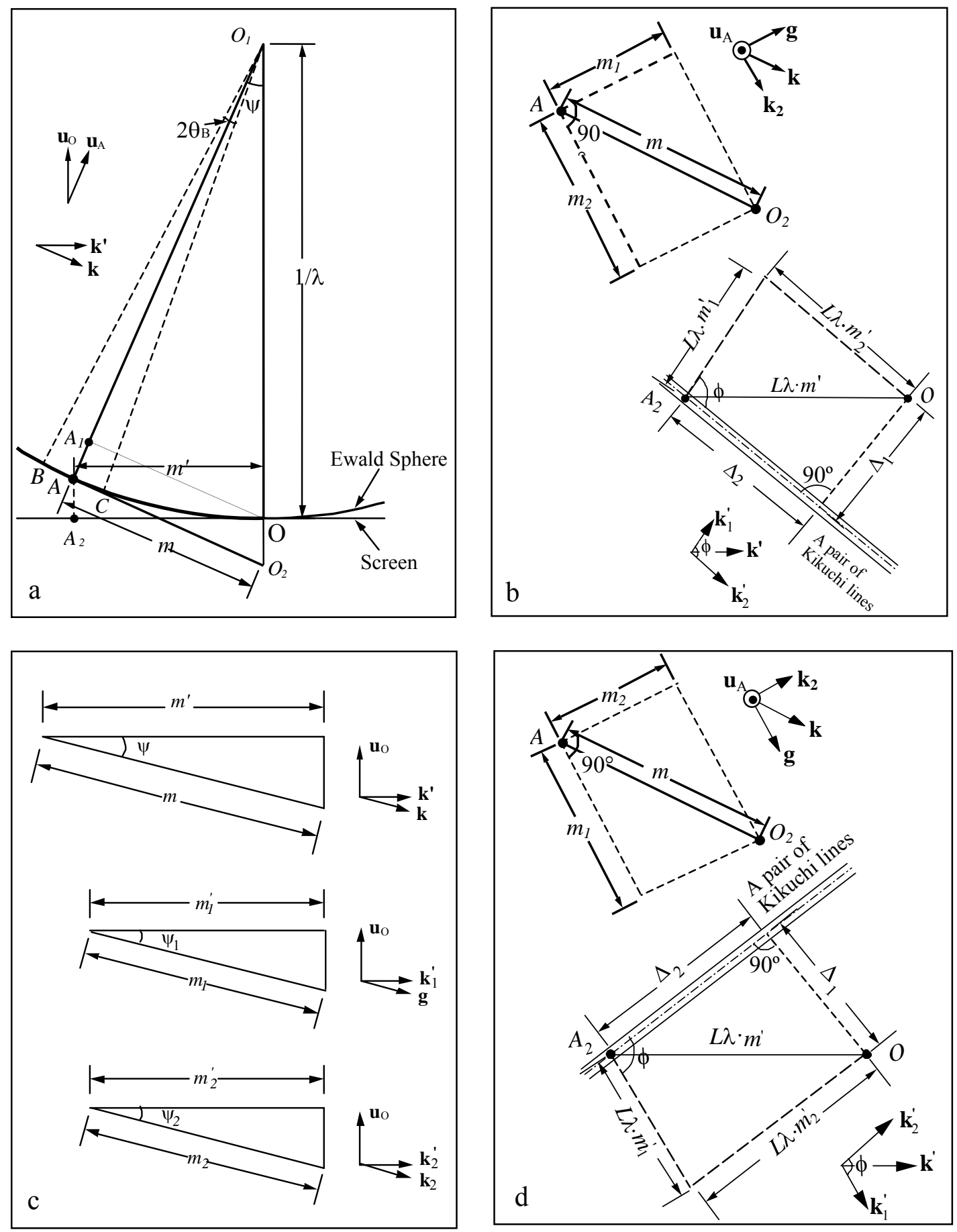

Fig. 1a A schematic diagram showing the geometry of Ewald sphere

Fig. 1b The top schematic diagram showing the relationship of $\mathbf{g}, \mathbf{k}$ and $\mathbf{k}_{2}\left(\mathbf{k}_{2}=\mathbf{g} \times \mathbf{u}_{\mathrm{A}}\right)$ in the plane perpendicular to $\mathbf{u}_{\mathrm{A}}$ and the bottom one showing the projected lines in the plane perpendicular to $\mathbf{u}_{\mathrm{O}}$

Fig. 1c Concise diagram shows the relations of dislocations in the planes perpendicular to $\mathbf{u}_{\mathrm{O}}$ and $\mathbf{u}_{\mathrm{A}}$

Fig. 1d similar to $1 b$, but $\mathbf{k}_{2}=\mathbf{u}_{\mathrm{A}} \times \mathbf{g}$ 
B
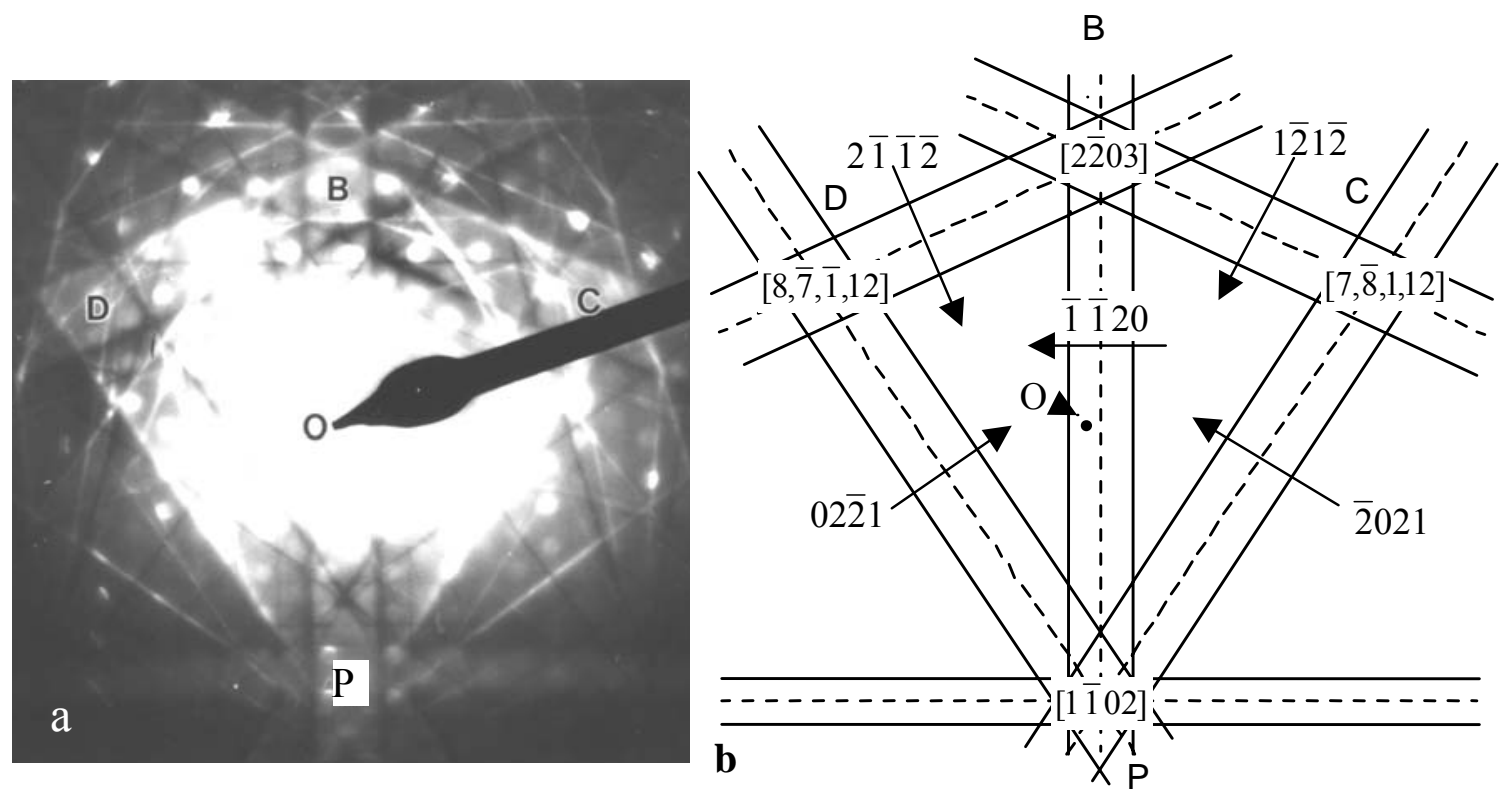

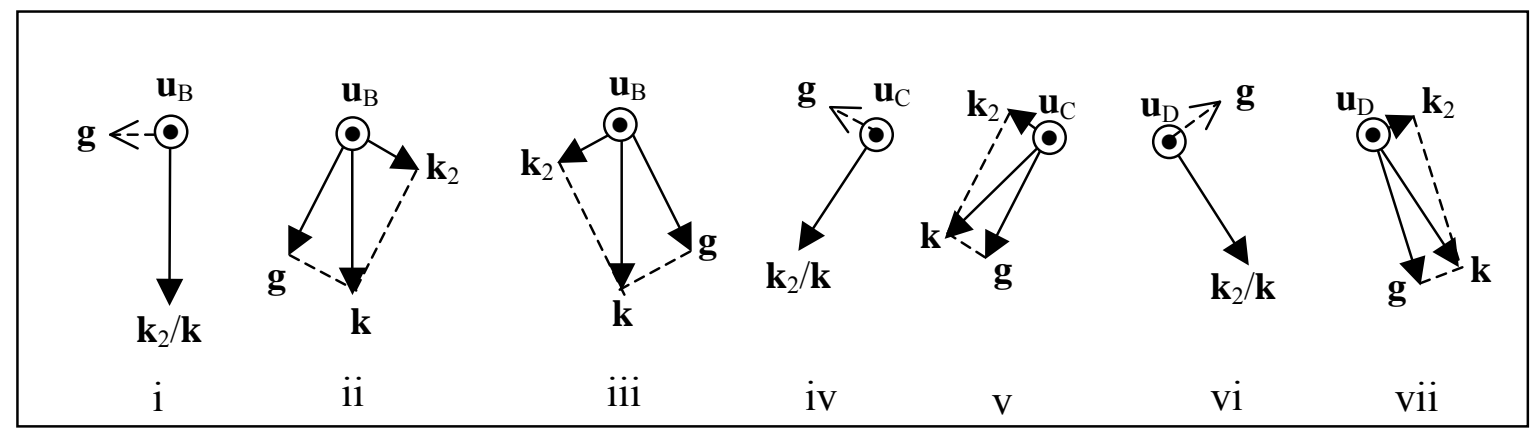

C

Fig. 2 The CBED pattern and the corresponding schematic program near [ $1 \overline{1} 02]$ of hexagonal titanium. (a) the recorded Kikuchi pattern; (b) illustration diagram corresponding to Fig (a); (c) choice of $\mathbf{k}_{2}$. Note the $\mathbf{g}$ vector in Figs. 2ci, 2civ and 2cvi can be chosen as opposite direction, but $\mathbf{k}_{2}$ has to be changed according to equation (8a) or $(8 b)$. 
Journal of Microscopy, Vol. 211, Pt 2 August 2003, pp. 130-136

Received 23 September 2002; Accepted 3 April 2003
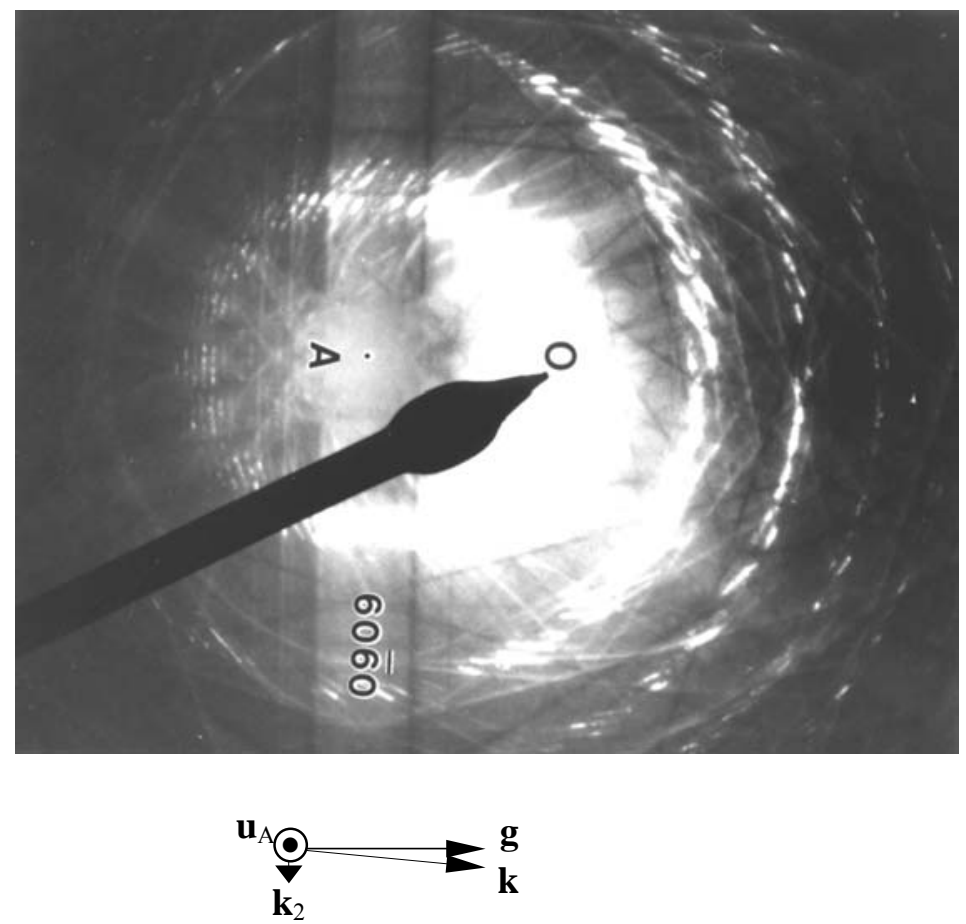

Fig. 3 The CBED pattern near $[2 \overline{4} 23]$ of $\mathrm{Ti}_{5} \mathrm{Si}_{3}$ phase 


\section{Appendix 1}

Fig. $1 \mathrm{~b}$ illustrates these vectors in the planes perpendicular to $\mathbf{u}_{\mathrm{O}}$ and $\mathbf{u}_{\mathrm{A}}$, thus

$$
\begin{aligned}
& \mathbf{k}_{1}^{\prime}=\frac{m_{1}}{m_{1}^{\prime}} \cdot \frac{\mathbf{g}}{|\mathbf{g}|}+\frac{\sqrt{m_{1}^{2}-\left(m_{1}^{\prime}\right)^{2}}}{m_{1}^{\prime}} \cdot \frac{\mathbf{u}_{\mathrm{O}}}{\left|\mathbf{u}_{\mathrm{O}}\right|} \\
& \mathbf{k}_{2}^{\prime}=\frac{m_{2}}{m_{2}^{\prime}} \cdot \frac{\mathbf{k}_{2}}{\left|\mathbf{k}_{2}\right|}+\frac{\sqrt{m_{2}^{2}-\left(m_{2}^{\prime}\right)^{2}}}{m_{2}^{\prime}} \cdot \frac{\mathbf{u}_{\mathrm{O}}}{\left|\mathbf{u}_{\mathrm{O}}\right|}
\end{aligned}
$$

From equations (A1, A2), it can be shown:

$$
\begin{aligned}
\mathbf{k}_{1}^{\prime} \cdot \mathbf{k}_{2}^{\prime}= & \frac{m_{1} \cdot m_{2}}{m_{1}^{\prime} \cdot m_{2}^{\prime}} \cdot \frac{\mathbf{g} \cdot \mathbf{k}_{2}}{\left|\mathbf{g} \cdot \mathbf{k}_{2}\right|}+\frac{\sqrt{m_{1}^{2}-\left(m_{1}^{\prime}\right)^{2}} \cdot \sqrt{m_{2}^{2}-\left(m_{2}^{\prime}\right)^{2}}}{m_{1}^{\prime} \cdot m_{2}^{\prime}} \cdot \frac{\mathbf{u}_{\mathrm{O}} \cdot \mathbf{u}_{\mathrm{O}}}{\left|\mathbf{u}_{\mathrm{O}} \cdot \mathbf{u}_{\mathrm{O}}\right|} \\
& +\frac{m_{1} \cdot \sqrt{m_{2}^{2}-\left(m_{2}^{\prime}\right)^{2}}}{m_{1}^{\prime} \cdot m_{2}^{\prime}} \cdot \frac{\mathbf{u}_{\mathrm{O}} \cdot \mathbf{g}}{\left|\mathbf{u}_{\mathrm{O}} \cdot \mathbf{g}\right|}+\frac{m_{2} \cdot \sqrt{m_{1}^{2}-\left(m_{1}^{\prime}\right)^{2}}}{m_{1}^{\prime} \cdot m_{2}^{\prime}} \cdot \frac{\mathbf{u}_{\mathrm{O}} \cdot \mathbf{k}_{2}}{\left|\mathbf{u}_{\mathrm{O}} \cdot \mathbf{k}_{2}\right|}
\end{aligned}
$$

Since $\mathbf{g}$ and $\mathbf{k}_{2}$ are perpendicular to each other, then

$$
\mathbf{g} \cdot \mathbf{k}_{2}=0, \text { and } \frac{\mathbf{u}_{\mathrm{O}} \cdot \mathbf{u}_{\mathrm{O}}}{\left|\mathbf{u}_{\mathrm{O}} \cdot \mathbf{u}_{\mathrm{O}}\right|}=1
$$

According to the geometrical diagram of Fig. 1c

and

$$
\begin{aligned}
& \frac{\mathbf{u}_{\mathrm{O}} \cdot \mathbf{g}}{\left|\mathbf{u}_{\mathrm{O}} \cdot \mathbf{g}\right|}=-\sin \left(\Psi_{1}\right)=-\frac{\sqrt{m_{1}^{2}-\left(m_{1}^{\prime}\right)^{2}}}{m_{1}} \\
& \frac{\mathbf{u}_{\mathrm{O}} \cdot \mathbf{k}_{2}}{\left|\mathbf{u}_{\mathrm{O}} \cdot \mathbf{k}_{2}\right|}=-\sin \left(\Psi_{2}\right)=-\frac{\sqrt{m_{2}^{2}-\left(m_{2}^{\prime}\right)^{2}}}{m_{2}}
\end{aligned}
$$

Thus equation (A3) can be simplified as

$\mathbf{k}_{1}^{\prime} \cdot \mathbf{k}_{2}^{\prime}=-\frac{\sqrt{m_{1}^{2}-\left(m_{1}^{\prime}\right)^{2}} \cdot \sqrt{m_{2}^{2}-\left(m_{2}^{\prime}\right)^{2}}}{m_{1}^{\prime} \cdot m_{2}^{\prime}}=-\left(\lambda \cdot m_{1}\right) \cdot\left(\lambda \cdot m_{2}\right)$

thus,

$$
\cos (\phi)=\mathbf{k}_{1}^{\prime} \cdot \mathbf{k}_{2}^{\prime}=-\frac{\Delta_{1} \cdot \Delta_{2}}{\sqrt{L^{2}-\Delta_{1}^{2}} \cdot \sqrt{L^{2}-\Delta_{2}^{2}}}
$$

